\title{
Three-year experience with immediate extubation in pediatric patients after congenital cardiac surgery
}

Christopher F. Tirotta ${ }^{{ }^{*}}$ DD, Stephen Alcos ${ }^{1}$, Richard G. Lagueruela', Daria Salyakina², Weize Wang ${ }^{2}$, Jessica Hughes ${ }^{1}$, Marysory Irizarry ${ }^{1}$ and Redmond P. Burke ${ }^{3}$

\begin{abstract}
Background: In pediatric cardiac anesthesiology, there is increased focus on minimizing morbidity, ensuring optimal functional status, and using health care resources sparingly. One aspect of care that has potential to affect all of the above is postoperative mechanical ventilation. Historically, postoperative ventilation was considered a must for maintaining patient stability. Ironically, it is recognized that mechanical ventilation may increase risk of adverse outcomes in the postoperative period. Hence, many institutions have advocated for immediate extubation or early extubation after many congenital heart surgeries which was first reported decades ago.
\end{abstract}

Methods: 637 consecutive patient charts were reviewed for pediatric patients undergoing cardiac surgery with cardiopulmonary bypass. Patients were placed into three groups. Those that were extubated in the operating room $(\mathrm{OR})$ at the conclusion of surgery (Immediate Extubation or IE), those that were extubated within six hours of admission to the ICU (Early Extubation or EE) and those that were extubated sometime after six hours (Delayed Extubation or DE). Multiple variables were then recorded to see which factors correlated with successful Immediate or Early Extubation.

Results: Overall, 338 patients (53.1\%) had IE), 273 (42.8\%) had DE while only 26 patients (4.1\%) had EE. The median age was 1174 days for the IE patients, 39 days for the DE patients, whereas 194 days for EE patients $(p<0.001)$. Weight and length were also significantly different in at least one extubation group from the other two $(p<0.001)$. The median ICU LOS was 3 and 4 days for IE and EE patients respectively, whereas it was 9.5 days for DE patients $(p<0.001)$. DE group had a significant longer median anesthesia time and cardiopulmonary bypass time than the other two extubation groups ( $p>63,826.88<0.001$ ). Regional low flow perfusion, deep hypothermia, deep hypothermic circulatory arrest, redo sternotomy, use of other sedatives, furosemide, epinephrine, vasopressin, open chest, cardiopulmonary support, pulmonary edema, syndrome, as well as difficult intubation were significantly associated with delayed extubation (IE, EE or DE).

Conclusions: Immediate and early extubation was significantly associated with several factors, including patient age and size, duration of CPB, use of certain anesthetic drugs, and the amount of blood loss and blood replacement. IE can be successfully accomplished in a majority of pediatric patients undergoing surgery for congenital heart disease, including in a minority of infants.

Keywords: Cardiac surgery, Congenital heart disease, Early extubation, Extubation, Pediatric

\footnotetext{
* Correspondence: christirotta@att.net

${ }^{1}$ Cardiac Anesthesia, Department of Anesthesiology, Nicklaus Children's

Hospital, Miami, USA

Full list of author information is available at the end of the article
}

(c) The Author(s). 2020 Open Access This article is distributed under the terms of the Creative Commons Attribution 4.0 International License (http://creativecommons.org/licenses/by/4.0/), which permits unrestricted use, distribution, and reproduction in any medium, provided you give appropriate credit to the original author(s) and the source, provide a link to the Creative Commons license, and indicate if changes were made. The Creative Commons Public Domain Dedication waiver (http://creativecommons.org/publicdomain/zero/1.0/) applies to the data made available in this article, unless otherwise stated. 


\section{Background}

Congenital heart disease (CHD) affects nearly $1 \%$ of - or about 40,000 - births per year in the United States [1]. About 25\% of babies with a CHD have critical CHD. Infants with critical CHD generally need surgery or other procedures in their first year of life, many of these surgeries requiring cardiopulmonary bypass. Moreover, nearly all of these surgeries and procedures require general anesthesia with endotracheal intubation. Thus, the anesthesia technique plays an integral role in improving patient outcomes after congenital cardiac surgery.

In pediatric cardiac anesthesiology, there is an increased attention focused on minimizing patient trauma with emphasis on minimizing morbidity, ensuring optimal functional status, and using health care resources sparingly. One aspect of care that has potential to affect all of the above is postoperative mechanical ventilation $[2,3]$. Historically, postoperative ventilation was considered a must for maintaining patient stability. Ironically, it is recognized that mechanical ventilation may increase the risk of adverse outcomes in the postoperative period [4]. Hence, many institutions have advocated for immediate extubation (IE) or early extubation (EE) after congenital heart surgeries which was first reported decades ago [5-7].

,IE/EE may translate into earlier enteral feed advancement and potentially a shorter hospital length of stay [8]. IE may also lessen the need for analgesic and sedative medications because endotracheal intubation can be a noxious stimulant. The association of prolonged endotracheal intubation with nosocomial infections, including ventilator-associated pneumonia, is well documented [3].

The rationale for conducting this study is to confirm that IE can be done safely in pediatric patients after congenital cardiac surgery with improved patient outcomes. The factors that are associated or correlated with successful IE will be analyzed; included will be both the anesthesia and non-anesthesia/surgical variables.

\section{Methods}

After receiving Institutional Review Board (IRB) exempt status from the Research Institute of Nicklaus Children's Hospital, we retrospectively reviewed the charts of all patients undergoing cardiac surgery for congenital heart disease between May 1, 2014 to June 30, 2017. These cases were done by one of three pediatric cardiac anesthesiologists and one of three cardiac surgeons; the cases were equally distributed between the anesthesiologists, but one surgeon performed over $90 \%$ of the surgeries. Patients were placed into three groups. Those that were extubated in the operating room (OR) at the conclusion of surgery (Immediate Extubation or IE), those that were extubated within six hours of admission to the ICU (Early Extubation or EE) and those that were extubated sometime after six hours (Delayed Extubation or DE). We then recorded the following variables:

Patient related factors like age at time of operation (days), sex of patient, weight and length $(\mathrm{kg}$ and $\mathrm{cm})$.

Surgical related factors like duration of: cardiopulmonary bypass time (CPB), aortic cross clamp time, regional low flow perfusion time (RLF), surgical time, anesthesia time, and time between end of surgery and room out (ES-RO). These also include lowest temperature achieved during surgery, use of deep hypothermia (low temp of less than $25^{\circ} \mathrm{C}$ ), duration deep hypothermic circulatory arrest (DHCA), and whether the surgery entailed a redo-sternotomy.

Transfusion related factors like volume of blood products used in the OR. These include packed red blood cells (PRBC) cell saver, fresh frozen plasma (FFP), plateletpheresis, cryoprecipitate, human fibrinogen concentrate (HFC), urine output, and estimated blood loss (EBL) in first $24 \mathrm{~h}$ post-op.

Anesthesia and pharmacological factors like the amount of Lasix, mannitol, and crystalloid used. Also, the need for inotropic support which includes the following: epinephrine, milrinone, and vasopressin. We looked at whether the drug was used and the amount. We recorded the amount of the following anesthetics: fentanyl, morphine, propofol, midazolam, other benzodiazepines (like lorazepam), other sedatives (like ketamine), dexmedetomidine, neostigmine, and sugammadex,

And finally other factors like: open chest on leaving the OR, need for Cardiopulmonary Support (CPS) leaving OR, identity of the anesthesiologist, length of Intensive Care Unit (ICU) stay, patient length of hospital stay (PLOS), difficult intubation, reintubation within $24 \mathrm{~h}$ of ICU arrival, and the presence of congenital birth syndromes.

\section{Statistical analysis methods}

Descriptive statistics were used to summarize characteristics of the study patients. Sub-group analysis was conducted among infants $\leq 1$ year of age. Frequencies and percentages were used to present categorical variables overall and stratified by extubation group (IE [immediate extubation], EE [early extubation], and DE [delayed extubation]). Sample median and interquartile range (IQR) of variables including age, weight, length, ICU length of stay (LOS), hospital LOS, intubation days, anesthesia time, CPB time, and time of ES-RO were calculated due to non-normal distribution of the data and were reported for overall and by extubation groups. To determine if there is a significant difference between extubation groups (IE, EE, or DE), fisher exact tests and Kruskal Wallis test was used for categorical and continuous variables respectively.

In order to understand whether there was a significant difference by extubation group in procedure duration, or the dose of an administered medication/blood product 
during the surgery, adjusted median regression was performed for mannitol, amount, and epineprhine respectively; Zero Truncated Poisson regression was used to predict intubation days, while log-normal regression was applied to predict other continuous variables. Extubation group (IE + EE vs. DE) was used as the main predictor, while age, weight, and length were adjusted as covariates. Patients with IE and EE were included as one group due to small sample size in the EE group. Blood products were measured at two times. Thus, generalized linear models with repeated measures was used to assess the effect of extubation group on the dose of each blood product overtime adjusting for age, weight, and length as covariates. For the administered medications/blood products and procedures, not every patient received these items. Thus, when we compare the doses of each medication/blood product, or duration of the procedure by the extubation groups, patients that did not receive the product/procedure were excluded from the lognormal regression and generalized linear models. For example, only 536 out of the 637 patients had their aorta cross clamped (XC) as part of the procedure; we conducted the regression analysis using the duration of $\mathrm{XC}$ from the 536 patients as the outcome.

Statistical analyses were performed by using the statistical software package SAS Enterprise Guide 7.1 (SAS Institute Inc., Cary, NC). All statistical analyses were performed at 0.05 level of significance.

\section{Results}

Six hundred thirty-seven cases are included in this analysis (Table 1). Overall, 338 patients (53.1\%) had IE, 273 (42.8\%) had DE, while only 26 patients (4.1\%) had EE. The median age was 1174 days for the IE patients, 39 days for the DE patients, whereas 194 days for EE patients $(p<0.001)$. Weight and length were also significantly different in at least one extubation group from the other two $(p<0.001)$. The median ICU LOS was three and four days for IE and EE patients respectively, whereas it was 9.5 days for DE patients $(\mathrm{p}<0.001)$. DE group had a significant longer median anesthesia time and $\mathrm{CPB}$ time in minutes than the other two extubation groups $(p<0.001)$. Median time for End of Surgery to Room Out (ES-RO) for EE was 16.5 min, whereas it was $14 \mathrm{~min}$ in both IE and DE groups $(p=0.029)$. RLF, deep hypothermia, DHCA, redo, use of other sedatives, lasix, epinephrine, vasopressin, open chest, CPS, pulmonary edema, syndrome, as well as difficult intubation were significantly associated with extubation time (IE, EE or DE) (Table 2, $p<0.05$ ).

Results suggest a significant an association between extubation group and aortic cross clamp time, the longer the cross-clamp time the more likely the patient would be in the DE group, controlled by patient's age, weight and length (Table $3, p<0.001$ ). Significant association was also found between extubation group with low temperature, urine output, doses of fentanyl, midazolam, rocuronium, human fibrinogen concentrate (HFC), milrinone, epinephrine, and ICU EBL, adjusting for patient's age, weight and length (Table $3, p<0.05$ ).

On average, a significant association was found between extubation group and the dose of PRBC in that patients with delayed extubation had a higher dose of PRBC $(p<0.001)$, controlling for age, weight and length. There was no significant difference in the amount of any other blood product.

Overall, of the total 637 patients, 350 (54.9\%) patients were infants (Table 4). Of the 350 patients, 232 (66.3\%) had DE, 103 (29.4\%) had IE, and 14 (4.3\%) had EE. The median age was 20 days for the DE patients, 174 days for the IE patients, and 157 days for EE patients $(p<0.0001)$. Weight and length were also significantly different in at least one extubation group from the other two $(\mathrm{p}<$ 0.0001). The youngest patient to successfully undergo IE was four days old and the smallest was $3.3 \mathrm{~kg}$. The median ICU LOS was 4 and 5 days for IE and EE patients

Table 1 Patient characteristic and times

\begin{tabular}{|c|c|c|c|c|c|c|c|c|c|}
\hline \multirow[t]{3}{*}{ Variable } & \multirow{2}{*}{\multicolumn{2}{|c|}{ Overall }} & \multicolumn{6}{|c|}{ Extubation } & \multirow[t]{3}{*}{$p^{b}$} \\
\hline & & & \multicolumn{2}{|l|}{$\mathrm{IE}$} & \multicolumn{2}{|l|}{$\mathrm{DE}$} & \multicolumn{2}{|c|}{$\mathrm{EE}$} & \\
\hline & $\mathrm{N}$ & $\begin{array}{l}\text { Median (Interquartile } \\
\text { Range) }\end{array}$ & $N$ & $\begin{array}{l}\text { Median (Interquartile } \\
\text { Range) }\end{array}$ & $\mathrm{N}$ & $\begin{array}{l}\text { Median (Interquartile } \\
\text { Range) }\end{array}$ & $\mathrm{N}$ & $\begin{array}{l}\text { Median (Interquartile } \\
\text { Range) }\end{array}$ & \\
\hline Age (days) & 637 & $236(1545)$ & 338 & 1174 (3193) & 273 & $39(181)$ & 26 & $194(1098)$ & $<0.001$ \\
\hline Weight (kg) & 637 & $7.1(12.6)$ & 338 & $14(24)$ & 273 & $3.5(2.9)$ & 26 & $7.5(8.8)$ & $<0.001$ \\
\hline Length $(\mathrm{cm})$ & 634 & $67(50)$ & 338 & $96(65)$ & 270 & $52(13)$ & 26 & $65(36)$ & $<0.001$ \\
\hline ICU LOS (days) & 635 & $5(7)$ & 337 & $3(4)$ & 272 & $9.5(15.0)$ & 26 & $4(5)$ & $<0.001$ \\
\hline PLOS (days) & 634 & $8(10)$ & 337 & $5(4)$ & 271 & $12(16)$ & 26 & $5.5(5)$ & $<0.001$ \\
\hline Anes time (min) & 637 & $262(110)$ & 338 & $247(90)$ & 273 & $298(117)$ & 26 & $230(111)$ & $<0.001$ \\
\hline CPB (min) & 635 & $99(73)$ & 337 & $84(44)$ & 272 & $138(94)$ & 26 & $71(31)$ & $<0.001$ \\
\hline Time (ES-RO) (min) & 635 & $14(7)$ & 337 & $14(8)$ & 272 & $14(7)$ & 26 & $17(8)$ & 0.029 \\
\hline
\end{tabular}

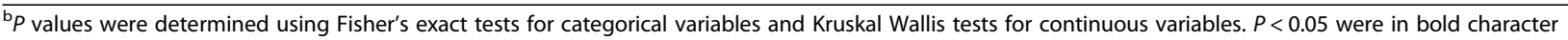


Table 2 Patients characteristics, medical conditions, and therapies $(N=637)$

\begin{tabular}{|c|c|c|c|c|c|}
\hline \multirow[t]{3}{*}{ Variable } & \multirow{2}{*}{$\begin{array}{l}\text { Overall } \\
(N=637)\end{array}$} & \multicolumn{3}{|c|}{ Extubation Time } & \multirow[t]{3}{*}{$p^{b}$} \\
\hline & & $\mathrm{IE}(N=338)$ & $\mathrm{DE}(N=273)$ & $\mathrm{EE}(N=26)$ & \\
\hline & $N\left(\%^{\mathrm{a}}\right)$ & $N\left(\%^{\mathrm{a}}\right)$ & $N\left(\%^{\mathrm{a}}\right)$ & $\mathrm{N}\left(\%^{\mathrm{a}}\right)$ & \\
\hline Sex & & & & & 0.584 \\
\hline Male & $349(54.8)$ & $184(54.4)$ & $153(56.0)$ & $12(46.2)$ & \\
\hline Female & $285(44.7)$ & $153(45.3)$ & $118(43.2)$ & $14(53.8)$ & \\
\hline Regional Low Perfusion & & & & & $<0.001$ \\
\hline Yes & $92(14.4)$ & $4(1.2)$ & $87(31.9)$ & $1(3.8)$ & \\
\hline No & $544(85.4)$ & $334(98.8)$ & $185(67.8)$ & $25(96.2)$ & \\
\hline Deep Hypothermia $(T e m p<25)$ & & & & & $<0.001$ \\
\hline Yes & $150(23.5)$ & $14(4.1)$ & $134(49.1)$ & $2(7.7)$ & \\
\hline No & $487(76.5)$ & $324(95.9)$ & $139(50.9)$ & $24(92.3)$ & \\
\hline DHCA & & & & & $<0.001$ \\
\hline Yes & $42(6.6)$ & $29(0.6)$ & $40(14.7)$ & $0(0.0)$ & \\
\hline No & $595(93.4)$ & $336(99.4)$ & $233(85.3)$ & $26(100.0)$ & \\
\hline Redo sternotomy & & & & & $<0.001$ \\
\hline Yes & $188(29.5)$ & $135(39.9)$ & $45(16.5)$ & $8(30.8)$ & \\
\hline No & $446(70.0)$ & $201(59.5)$ & $228(83.5)$ & $17(65.4)$ & \\
\hline \multicolumn{6}{|l|}{ Other Benzodiazepines } \\
\hline No & $636(99.9)$ & $337(99.7)$ & $273(100.0)$ & $26(100.0)$ & NA \\
\hline Other Sedatives & & & & & 0.004 \\
\hline Yes & $45(7.1)$ & $14(4.1)$ & $30(11.0)$ & $1(3.8)$ & \\
\hline No & $591(92.8)$ & $324(95.9)$ & $242(88.6)$ & $25(96.2)$ & \\
\hline Lasix & & & & & 0.004 \\
\hline Yes & $563(88.4)$ & $310(91.7)$ & $230(84.2)$ & $23(88.5)$ & \\
\hline No & $43(6.8)$ & $13(3.8)_{-}$ & $29(10.6)$ & $1(3.8)$ & \\
\hline Factor VII & & & & & NA \\
\hline No & $637(99.5)$ & $338(100)$ & $273(100.0)$ & $26(100.0)$ & \\
\hline Milrinone & & & & & 0.689 \\
\hline Yes & $633(99.4)$ & 335 (99.1) & $272(99.6)$ & $26(100.0)$ & \\
\hline No & $4(0.6)$ & $3(0.9)$ & $1(0.4)$ & $0(0.0)$ & \\
\hline Epinephrine & & & & & $<0.001$ \\
\hline Yes & $155(24.3)$ & $8(2.4)$ & $144(52.7)$ & 3 & \\
\hline No & $482(75.7)$ & $330(97.6)$ & $129(47.3)$ & 23 & \\
\hline Vasopresin & & & & & 0.008 \\
\hline Yes & $7(1.1)$ & $0(0.0)$ & $7(2.6)$ & $0(0.0)$ & \\
\hline No & $630(98.9)$ & $338(100.0)$ & $266(97.4)$ & $26(100.0)$ & \\
\hline Open chest & & & & & $<0.001$ \\
\hline Yes & $108(17.0)$ & $0(0.0)$ & 108 (39.6) & $0(0.0)$ & \\
\hline No & $529(83)$ & $338(100.0)$ & $165(60.4)$ & $26(100.0)$ & \\
\hline Cardiopulmonary Support & & & & & $<0.001$ \\
\hline Yes & $20(3.1)$ & $0(0.0)$ & $20(7.3)$ & $0(0.0)$ & \\
\hline No & $617(96.9)$ & $338(100.0)$ & $253(92.7)$ & $26(100.0)$ & \\
\hline Pulmonary Edema & & & & & 0.001 \\
\hline Yes & $13(2.0)$ & $1(0.3)$ & $12(4.4)$ & $0(0.0)$ & \\
\hline
\end{tabular}


Table 2 Patients characteristics, medical conditions, and therapies ( $N=637)$ (Continued)

\begin{tabular}{|c|c|c|c|c|c|}
\hline \multirow[t]{3}{*}{ Variable } & \multirow{2}{*}{$\begin{array}{l}\text { Overall } \\
(N=637)\end{array}$} & \multicolumn{3}{|c|}{ Extubation Time } & \multirow[t]{3}{*}{$p^{b}$} \\
\hline & & $\mathrm{IE}(N=338)$ & $\mathrm{DE}(N=273)$ & $\mathrm{EE}(N=26)$ & \\
\hline & $N\left(\%^{\mathrm{a}}\right)$ & $N\left(\%^{a}\right)$ & $N\left(\%^{\mathrm{a}}\right)$ & $N\left(\%^{\mathrm{a}}\right)$ & \\
\hline No & $624(98.0)$ & $337(99.7)$ & $261(95.6)$ & $26(100.0)$ & \\
\hline Reintubation within $24 \mathrm{~h}$ & & & & & 0.100 \\
\hline Yes & $17(2.7)$ & $5(1.5)$ & $11(4.0)$ & $1(3.8)$ & \\
\hline No & $617(96.9)$ & $331(97.9)$ & $261(95.6)$ & $25(96.2)$ & \\
\hline Syndrome & & & & & $<0.001$ \\
\hline Yes & $113(17.7)$ & $34(10.1)$ & $74(27.1)$ & $5(19.2)$ & \\
\hline No & $519(81.5)$ & $302(89.3)$ & $196(71.8)$ & $21(80.8)$ & \\
\hline Difficult intubation & & & & & 0.001 \\
\hline Yes & $13(2.0)$ & $1(0.3)$ & 12 & $0(0.0)$ & \\
\hline No & $618(97.0)$ & $333(98.5)$ & $259(94.9)$ & $26(100.0)$ & \\
\hline
\end{tabular}

Note. ${ }^{\mathrm{a}} \% \mathrm{~s}$ are column percentages except the first row. The sum of $\% \mathrm{~s}$ of a variable may not be 100 due to missing values

${ }^{\mathrm{b}} P$ values were determined using Fisher's exact tests for categorical variables and Kruskal Wallis tests for continuous variables. $P<0.05$ were in bold character

Table 3 Descriptive statistics of procedures and administered medications with results of regression analyses

\begin{tabular}{|c|c|c|c|c|c|c|c|c|c|}
\hline \multirow[t]{3}{*}{ Variable $^{a}$} & \multicolumn{2}{|c|}{$\begin{array}{l}\text { Underwent Treatment/ } \\
\text { Procedure }\end{array}$} & \multicolumn{6}{|c|}{ Patients Underwent the Treatment/Procedure } & \multirow[b]{3}{*}{$p^{b}$} \\
\hline & \multirow{2}{*}{$\begin{array}{l}\text { Yes } \\
\text { N (\%) }\end{array}$} & \multirow{2}{*}{$\begin{array}{l}\text { No } \\
\text { N (\%) }\end{array}$} & \multicolumn{2}{|c|}{ Overall } & \multicolumn{2}{|c|}{ Delayed Extubation } & \multicolumn{2}{|c|}{ Immediate/Early Extubation } & \\
\hline & & & $\bar{N}$ & Median (Qrange) & $\bar{N}$ & Median (Qrange) & $\bar{N}$ & Median (Qrange) & \\
\hline Aortic cross clamp (min) & $536(84)$ & $101(16)$ & 536 & $58.4(48.0)$ & 249 & $77.0(62.0)$ & 287 & $50.0(33.0)$ & $<0.001$ \\
\hline Low Temperature $\left({ }^{\circ} \mathrm{C}\right)$ & $630(98.9)$ & $7(1.1)$ & 630 & $29.1(6.5)$ & 270 & $24.0(12.0)$ & 360 & $30.6(4.50)$ & $<0.001$ \\
\hline DHCA (min) & $42(6.6)$ & $5.95(93.4)$ & 42 & $25(33.0)$ & 40 & $21.0(33.5)$ & 2 & $26.5(1.00)$ & 0.662 \\
\hline Urine (cc/kg) & $616(96.7)$ & $21(3.3)$ & 616 & $9.51(14.2)$ & 254 & $6.47(9.74)$ & 362 & $12.7(16.3)$ & 0.024 \\
\hline Fentanyl (mcg/kg) & $606(95.1)$ & $31(4.9)$ & 606 & $10.26(16.6)$ & 269 & $22.7(18.9)$ & 337 & $5.32(5.44)$ & $<0.001$ \\
\hline Morphine (mg/kg) & $42(6.6)$ & $595(93.4)$ & 42 & $0.22(0.16)$ & 7 & $0.22(0.23)$ & 35 & 0.23 & NA \\
\hline Propofol (mg/kg) & $478(75.0)$ & $159(25.0)$ & 478 & $2.82(3.2)$ & 176 & $2.86(3.85)$ & 302 & $2.81(2.75)$ & 0.875 \\
\hline Dexmedetomidine (mcg/kg) & $586(92)$ & $51(8.0)$ & 586 & $2.88(2.3)$ & 229 & $3.03(2.58)$ & 357 & $2.80(1.99)$ & 0.240 \\
\hline Rocuronium (mg/kg) & 636 (99.8) & $1(0.2)$ & 636 & $3.13(2.0)$ & 272 & $4.07(2.60)$ & 364 & $2.72(1.34)$ & 0.019 \\
\hline Neostigmine (mg/kg) & $277(43.5)$ & $360(56.5)$ & 277 & $0.07(0.01)$ & 21 & $0.07(0.02)$ & 256 & $0.07(0.01)$ & 0.653 \\
\hline Sugammadex (mg/kg) & $97(15.2)$ & $540(84.8)$ & 97 & $4.35(2.2)$ & 7 & $4.35(1.02)$ & 90 & $4.37(2.34)$ & 0.079 \\
\hline Midazolam (mg/kg) & $153(24.0)$ & $484(76.0)$ & 153 & $0.09(0.1)$ & 29 & $0.20(0.25)$ & 124 & $0.08(0.09)$ & $<0.001$ \\
\hline Other Sedatives (mg/kg) & $48(7.5)$ & $589(92.5)$ & 48 & $6.48(10.8)$ & 31 & $4.63(3.27)$ & 17 & $15.0(0.79)$ & 0.242 \\
\hline Mannitol (mg/kg) & 405 (63.6) & $232(36.4)$ & 405 & $400(109.0)$ & 165 & 406. (100.) & 240 & $400(213)$ & 0.298 \\
\hline Lasix (mg/kg) & $564(88.5)$ & $73(11.5)$ & 564 & $0.42(0.4)$ & 231 & $0.59(0.32)$ & 333 & $0.31(0.25)$ & 0.997 \\
\hline NS Amount (cc/kg) & $631(99.1)$ & $6(0.9)$ & 631 & $17.77(16.8)$ & 268 & $20.6(15.9)$ & 363 & $16.2(15.6)$ & 0.669 \\
\hline $\mathrm{HFC}(\mathrm{mg} / \mathrm{kg})$ & $400(62.8)$ & $237(37.2)$ & 400 & $71.1(70.0)$ & 222 & 136. (74.7) & 178 & $70.0(3.97)$ & 0.008 \\
\hline Milrinone (mg/kg) & $635(99.7)$ & $2(0.3)$ & 635 & $0.15(0.04)$ & 273 & $0.16(0.06)$ & 362 & $0.14(0.03)$ & $<0.001$ \\
\hline Epinephrine (mg/kg) & $1.57(24.7)$ & $480(75.4)$ & 157 & $0.01(0.01)$ & 146 & $0.01(0.01)$ & 11 & $0.00(0.01)$ & 0.046 \\
\hline Vasopresin (mcg/kg) & $7(1.1)$ & $630(98.9)$ & 7 & $0.08(0.14)$ & 7 & $0.08(0.14)$ & 0 & NA & NA \\
\hline ICU EBL (cc/kg) & $612(96.1)$ & $25(3.9)$ & 612 & $21.5(28.5)$ & 260 & $31.0(43.5)$ & 352 & $15.0(19.0)$ & 0.026 \\
\hline Intubation (days) ${ }^{\#}$ & $296(46.5)$ & $341(53.5)$ & 632 & $0(3.0)$ & 270 & $3.00(4.00)$ & 362 & $0.00(0.00)$ & $<0.001$ \\
\hline
\end{tabular}

Note. ${ }^{a} 0 \mathrm{~s}$ in variables are excluded. \# $0 \mathrm{~s}$ were NOT Excluded (Intubation Days)

${ }^{\mathrm{b}} \mathrm{P}$-values are determined from median regression for Mannitol, Amount, and Epi; Poisson regression for Intubation days, and Log-Normal regression for other continuous variables. Extubation group (IE + EE vs. DE) was used as the main predictor adjusting for age, weight, and length as covariates. NAs are due to limited sample size. $P<0.05$ are in bold character 
Table 4 Patient characteristic and times

\begin{tabular}{|c|c|c|c|c|c|c|c|c|c|}
\hline \multirow[t]{3}{*}{ Variable } & \multirow{2}{*}{\multicolumn{2}{|c|}{ Overall }} & \multicolumn{6}{|c|}{ Extubation } & \multirow[t]{3}{*}{$p^{b}$} \\
\hline & & & \multicolumn{2}{|l|}{$\overline{\mathrm{DE}}$} & \multicolumn{2}{|l|}{$\mathrm{IE}$} & \multicolumn{2}{|l|}{ EE } & \\
\hline & $\bar{N}$ & $\begin{array}{l}\text { Median (Interquartile } \\
\text { Range) }\end{array}$ & $\mathrm{N}$ & $\begin{array}{l}\text { Median (Interquartile } \\
\text { Range) }\end{array}$ & $\bar{N}$ & $\begin{array}{l}\text { Median (Interquartile } \\
\text { Range) }\end{array}$ & $\bar{N}$ & $\begin{array}{l}\text { Median (Interquartile } \\
\text { Range) }\end{array}$ & \\
\hline Age (days) & 350 & $94(164.0)$ & 232 & $20(97.0)$ & 103 & $174(82.0)$ & 15 & $157(38.0)$ & $<0.0001$ \\
\hline Weight (kg) & 350 & $4.45(3.0)$ & 232 & $3.4(1.62)$ & 103 & $6.3(1.6)$ & 15 & $6.4(1.7)$ & $<0.0001$ \\
\hline Length $(\mathrm{cm})$ & 347 & $56(14.0)$ & 229 & $51(9.0)$ & 103 & $64(6.5)$ & 15 & $62(4.0)$ & $<0.0001$ \\
\hline ICU LOS (days) & 348 & $8(11.0)$ & 231 & $11(15.0)$ & 102 & $4(4.0)$ & 15 & $5(6.0)$ & $<0.0001$ \\
\hline PLOS (days) & 347 & $9(14.0)$ & 230 & $13(18.0)$ & 102 & $6(4.0)$ & 15 & $6(5.0)$ & $<0.0001$ \\
\hline Intubation (days) & 346 & $2(4.0)$ & 229 & $3(3.0)$ & 102 & $0(0.0)$ & 15 & $4(4.0)$ & $<0.0001$ \\
\hline Anes time (min) & 350 & $258.5(99.0)$ & 232 & $285(111.5)$ & 103 & $235(56.0)$ & 15 & $228(72.0)$ & $<0.0001$ \\
\hline CPB (min) & 350 & $104(74.0)$ & 232 & $134(88.5)$ & 103 & $80(30.0)$ & 15 & $80(28.0)$ & $<0.0001$ \\
\hline Time (ES-RO)(min) & 348 & $14(8.0)$ & 231 & $14(7.0)$ & 102 & $15(7.0)$ & 15 & $18(9.0)$ & 0.0126 \\
\hline
\end{tabular}

${ }^{\mathrm{b}} P$-values are determined using Fisher's exact tests for categorical variables and Kruskal Wallis tests for continuous variables. $P<0.05$ are in bold character. NAs are due to limited sample size. $P<0.05$ are in bold character

respectively, whereas it was 11 days for DE patients $(p<$ 0.0001). When compared to IE and EE, DE group had a significant longer median anesthesia time (285 vs 235[IE], $228[\mathrm{EE}]$ minutes) and CPB time (134 vs 80 [IE \&EE] minutes) $(p>0.0001)$. Median time (ES-RO) was $14 \mathrm{~min}$ for DE patients, $15 \mathrm{~min}$ for IE patients, and $18 \mathrm{~min}$ for EE patients $(p=0.0126)$. RLP, deep hypothermmia, DHCA, redo sternotomy, use of other sedatives, epinephrine, open chest, and CPS were significantly associated with extubation time (IE, EE or DE) (Table 5, $p<0.05$ ).

Adjusting for patient's age, weight, and length suggested that there was a significant association observed between extubation group and $\mathrm{XC}$, with longer $\mathrm{XC}$ times correlated with the DE group (Table $6, p<0.0001$ ). Similarly, significant association was found between extubation groups with low temperature, urine output, doses of fentanyl, dexmedetomidine, lasix, and crystalloid amount, adjusting for patient's age, weight and length (Table 6, $p<0.05$ ).

On average, a significant association was found between extubation group and the dose of PRBC in that patients with delayed extubation had a higher dose of PRBC ( $p=0.006)$, controlling for age, weight and length. There was no significant difference in the amount of any other blood product.

The case mix for the IE/EE groups and the DE group are listed in Tables 7 and 8.

\section{Discussion}

Our retrospective analysis demonstrated that a majority of older infants and pediatric congenital heart surgery patients can be successfully extubated in the operating room at the conclusion of surgery. The reintubation rate is less than $3 \%(2.7 \%)$ in all patients and the IE group actually had a lower reintubation rate $(1.5 \%)$ than the EE (3.8\%) or DE $(4.0 \%)$ groups that were extubated by the ICU staff hours or days after surgery. The reintubation rate for the infants was slightly higher, but still less than $4 \%$. There was no statistical difference between the groups. This is much better than the reported $11 \%$ (range 5 to $22 \%$ ) reintubation rate in the Pediatric Cardiac Critical Care Consortium multicenter study of neonates after cardiac surgery [9] who were extubated in the ICU hours or days later. However, our data was not restricted to neonates, who have the lowest IE/EE rate of all the patients studied.

Other relevant clinical studies have shown that most children undergoing congenital heart surgery can be extubated in the operating room. Many neonates, including those undergoing complex procedures, can be extubated within the first $24 \mathrm{~h}$ after surgery. Early extubation (EE) has been associated with low morbidity rates and shorter lengths of intensive care unit and hospital stays [10]. In additional studies, immediate extubation was associated with a shorter Intensive Care Unit (ICU) length of stay (LOS), lower postoperative ICU costs, and minimal increase in operating room turnover time, but without an increase in reintubation rates [6]. Even though costs were not analyzed in this review, our data also demonstrated reduced LOS in the CICU and LOS in the hospital without an increase in reintubation rates. Low gestational age, preoperative ventilatory support requirement, and prolonged cardiopulmonary bypass time were inversely associated with the ability to accomplish IE $[11,12]$. In a five-year cohort study of infants post repair for transposition of great arteries, greater $\mathrm{CPB}$ and cross-clamp times and minimum temperatures less than or equal to 30.4 degrees centigrade were associated with a lesser likelihood of IE [13]. Another five-year study demonstrated an inverse relationship between age and CPB time and early extubation [14].

Our analysis corroborates the findings listed above. The patients who experienced IE or EE had a much 
Table 5 Patients characteristics, medical conditions, and therapies $(N=350)$

\begin{tabular}{|c|c|c|c|c|c|}
\hline \multirow[t]{3}{*}{ Variable } & \multirow{3}{*}{$\begin{array}{l}\text { Overall } \\
(N=350) \\
N\left(\%^{a}\right)\end{array}$} & \multicolumn{3}{|c|}{ Extubation Time } & \multirow[t]{3}{*}{$p^{b}$} \\
\hline & & $\overline{\mathrm{DE}}(N=232)$ & $\mathrm{IE}(N=103)$ & $\mathrm{EE}(N=15)$ & \\
\hline & & $\mathrm{N}\left(\%^{\mathrm{a}}\right)$ & $N\left(\%^{\mathrm{a}}\right)$ & $N\left(\%^{a}\right)$ & \\
\hline Sex & & & & & 0.2433 \\
\hline Male & $206(58.9)$ & $136(58.6)$ & $64(62.1)$ & $6(40.0)$ & \\
\hline Female & $141(40.3)$ & $94(40.5)$ & $38(36.9)$ & $9(60.0)$ & \\
\hline Regional Low Perfusion & & & & & $<0.0001$ \\
\hline Yes & $88(25.1)$ & 85 (36.6) & $3(2.9)$ & . & \\
\hline No & $261(74.6)$ & $146(62.9)$ & $100(97.1)$ & $15(100.0)$ & \\
\hline Deep Hypothermia $(T e m p<25)$ & & & & & $<0.0001$ \\
\hline Yes & $127(36.3)$ & $122(52.6)$ & $4(3.9)$ & $1(6.7)$ & \\
\hline No & $223(63.7)$ & $110(47.4)$ & $99(96.1)$ & $14(93.3)$ & \\
\hline DHCA & & & & & $<0.0001$ \\
\hline Yes & $42(12.0)$ & $40(17.2)$ & $2(1.9)$ & . & \\
\hline No & $308(88.0)$ & $192(82.8)$ & $101(98.1)$ & $15(100.0)$ & \\
\hline Redo sternotomy & & & & & $<0.0001$ \\
\hline Yes & $66(18.9)$ & $27(11.6)$ & $34(33.0)$ & $5(33.3)$ & \\
\hline No & $282(80.6)$ & $205(88.4)$ & $68(66.0)$ & $9(60.0)$ & \\
\hline Other Benzodiasepines & & & & & NA \\
\hline No & $350(100.0)$ & $232(100.0)$ & $103(100.0)$ & $15(100.0)$ & \\
\hline Other Sedatives & & & & & 0.0026 \\
\hline Yes & $30(8.6)$ & $28(12.1)$ & $2(1.9)$ & . & \\
\hline No & $319(91.1)$ & $203(87.5)$ & $101(98.1)$ & $15(100.0)$ & \\
\hline Lasix & & & & & 0.1274 \\
\hline Yes & $33(9.4)$ & 27 (11.6) & $5(4.9)$ & $1(6.7)$ & \\
\hline No & $20(5.7)$ & $11(4.7)$ & $7(6.8)$ & $2(13.3)$ & \\
\hline Factor VII & & & & & NA \\
\hline No & $350(100.0)$ & $232(100.0)$ & $103(100.0)$ & $15(100.0)$ & \\
\hline Milrinone & & & & & 0.5613 \\
\hline Yes & $348(99.4)$ & 231 (99.6) & $102(99.0)$ & $15(100.0)$ & \\
\hline No & $2(0.6)$ & $1(0.4)$ & $1(1.0)$ & . & \\
\hline Epinephrine & & & & & $<0.0001$ \\
\hline Yes & 135 (38.6) & $132(56.9)$ & $1(1.0)$ & $2(13.3)$ & \\
\hline No & $215(61.4)$ & $100(43.1)$ & $102(99.0)$ & $13(86.7)$ & \\
\hline Vasopresin & & & & & 0.3729 \\
\hline Yes & $6(1.7)$ & $6(2.6)$ & & & \\
\hline No & $344(98.3)$ & $226(97.4)$ & $103(100.0)$ & $15(100.0)$ & \\
\hline Open chest & & & & & $<0.0001$ \\
\hline Yes & $101(28.9)$ & $101(43.5)$ & & & \\
\hline No & $249(71.1)$ & $131(56.5)$ & $103(100.0)$ & $15(100.0)$ & \\
\hline Cardiopulmonary support & & & & & 0.0023 \\
\hline Yes & $19(5.4)$ & $19(8.2)$ & & & \\
\hline No & $331(94.6)$ & $213(91.8)$ & $103(100.0)$ & $15(100.0)$ & \\
\hline Pulmonary Edema & & & & & 0.1812 \\
\hline Yes & $13(3.7)$ & $12(5.2)$ & $1(1.0)$ & . & \\
\hline
\end{tabular}


Table 5 Patients characteristics, medical conditions, and therapies $(N=350)$ (Continued)

\begin{tabular}{|c|c|c|c|c|c|}
\hline \multirow[t]{3}{*}{ Variable } & \multirow{3}{*}{$\begin{array}{l}\text { Overall } \\
(N=350) \\
N\left(\%{ }^{a}\right)\end{array}$} & \multicolumn{3}{|c|}{ Extubation Time } & \multirow[t]{3}{*}{$p^{b}$} \\
\hline & & $\mathrm{DE}(N=232)$ & $\mathrm{IE}(N=103)$ & $\mathrm{EE}(N=15)$ & \\
\hline & & $\mathrm{N}\left(\%^{\mathrm{a}}\right)$ & $\mathrm{N}\left(\%^{\mathrm{a}}\right)$ & $N\left(\%^{\mathrm{a}}\right)$ & \\
\hline No & $337(96.3)$ & $220(94.8)$ & $102(99.0)$ & $15(100.0)$ & \\
\hline Reintubation within $24 \mathrm{~h}$ & & & & & 1.000 \\
\hline Yes & $12(3.4)$ & $9(3.9)$ & $3(2.9)$ & . & \\
\hline No & $336(96.0)$ & $222(95.7)$ & $99(96.1)$ & $15(100.0)$ & \\
\hline Syndrome & & & & & 0.1183 \\
\hline Yes & $76(21.7)$ & $58(25.0)$ & $16(15.5)$ & $2(13.3)$ & \\
\hline No & $270(77.1)$ & $171(73.7)$ & $86(83.5)$ & $13(86.7)$ & \\
\hline Difficult intubation & & & & & 0.0883 \\
\hline Yes & $10(2.9)$ & $10(4.3)$ & & & \\
\hline No & $336(96.0)$ & $220(94.8)$ & $101(98.1)$ & $15(100.0)$ & \\
\hline
\end{tabular}

Note. ${ }^{a} \%$ s are column percentages except the first row. The sum of \%s of a variable may not be 100 due to missing values

b $P$-values are determined using Fisher's exact tests for categorical and Kruskal Wallis tests for continuous variables. $P<0.05$ are in bold character. NAs are due to limited sample size. $P<0.05$ are in bold character

Table 6 Descriptive statistics of procedures and administered medications with results of regression analyses

\begin{tabular}{|c|c|c|c|c|c|c|c|c|c|}
\hline \multirow[t]{3}{*}{ Variable $^{a}$} & \multicolumn{2}{|c|}{ Underwent Treatment/Procedure } & \multicolumn{6}{|c|}{ Patients Underwent the Treatment/Procedure } & \multirow[t]{3}{*}{$p^{b}$} \\
\hline & \multirow{2}{*}{$\begin{array}{l}\text { Yes } \\
\text { N (\%) }\end{array}$} & \multirow{2}{*}{$\begin{array}{l}\text { No } \\
\text { N (\%) }\end{array}$} & \multicolumn{2}{|c|}{ Overall } & \multicolumn{2}{|c|}{ Delayed Extubation } & \multicolumn{2}{|c|}{$\begin{array}{l}\text { Immediate/Early } \\
\text { Extubation }\end{array}$} & \\
\hline & & & $\mathrm{N}$ & Median (Qrange) & $\mathrm{N}$ & Median (Qrange) & $\mathrm{N}$ & Median (Qrange) & \\
\hline Aortic cross clamp (min) & $293(83.7)$ & $57(16.3)$ & 293 & $62.0(52.5)$ & 211 & $72.0(60.9)$ & 82 & & $<0.0001$ \\
\hline Low Temperature $\left({ }^{\circ} \mathrm{C}\right)$ & $346(98.9)$ & $4(1.1)$ & 346 & $28.3(13.3)$ & 230 & $18.9(12.0)$ & 116 & $30.2(4.9)$ & $<0.0001$ \\
\hline DHCA (min) & $42(12.0)$ & $308(88.0)$ & 42 & $25.0(33.0)$ & 40 & $21.0(33.5)$ & 2 & $26.5(1.00)$ & 0.662 \\
\hline Urine (cc/kg) & $330(94.3)$ & $20(5.7)$ & 330 & $5.7(8.6)$ & 214 & $5.1(7.6)$ & 116 & $7.43(10.3)$ & 0.002 \\
\hline Fentanyl (mcg/kg) & $347(99.1)$ & $3(0.9)$ & 347 & $17.9(21.7)$ & 232 & $27.0(17.4)$ & 115 & $8.82(10.7)$ & $<0.0001$ \\
\hline Morphine (mg/kg) & $4(1.1)$ & $346(98.9)$ & 4 & $0.4(2.0)$ & 1 & $0.16(0.00)$ & 3 & $0.56(3.60)$ & NA \\
\hline Propofol (mg/kg) & $246(70.3)$ & $104(29.7)$ & 246 & $3.0(3.6)$ & 150 & $2.8(4.27)$ & 96 & $3.08(3.0)$ & 0.594 \\
\hline Dexmedetomidine (mcg/kg) & $302(86.3)$ & $48(13.7)$ & 302 & $3.0(2.5)$ & 188 & $2.9(2.70)$ & 114 & $3.33(2.2)$ & 0.005 \\
\hline Roc uranium (mg/kg) & $349(99.7)$ & $1(0.3)$ & 349 & $3.5(2.6)$ & 231 & $4.2(2.7)$ & 118 & $2.73(1.0)$ & 0.742 \\
\hline Neostigmine (mg/kg) & $94(26.9)$ & $256(73.1)$ & 94 & $0.01(0.01)$ & 13 & $0.1(0.04)$ & 81 & $0.07(0.0)$ & 0.944 \\
\hline Sugammadex (mg/kg) & $35(10.0)$ & $315(90.0)$ & 35 & $5.0(4.1)$ & 5 & $4.4(1.00)$ & 30 & $5.37(4.1)$ & NA \\
\hline Midazolam (mg/kg) & $21(6.0)$ & $329(94.0)$ & 21 & $0.3(0.1)$ & 12 & $0.3(0.41)$ & 9 & $0.33(0.1)$ & 0.676 \\
\hline Other Sedatives (mg/kg) & $32(9.1)$ & $318(90.9)$ & 32 & $4.7(3.2)$ & 29 & $4.6(2.4)$ & 3 & $10.0(18.0)$ & NA \\
\hline Mannitol (mg/kg) & $218(62.3)$ & $132(37.7)$ & 218 & $407.2(100.0)$ & 148 & $409.0(100.0)$ & 70 & $406.0(100.0)$ & 0.661 \\
\hline Lasix (mg/kg) & $298(85.1)$ & $52(14.9)$ & 298 & $0.5(0.4)$ & 195 & $0.6(0.29)$ & 103 & $0.3(0.2)$ & 0.008 \\
\hline NS Amount (cc/kg) & $344(98.3)$ & $6(1.7)$ & 344 & $20.0(14.6)$ & 227 & $21.4(15.9)$ & 117 & $17.5(11.5)$ & 0.003 \\
\hline $\mathrm{HFC}(\mathrm{mg} / \mathrm{kg})$ & $300(85.7)$ & $50(14.3)$ & 300 & $98.7(71.0)$ & 208 & $137.0(75.8)$ & 92 & $70.1(55.5)$ & 0.129 \\
\hline Milrinone (mg/kg) & $349(99.7)$ & $1(0.3)$ & 349 & $0.2(0.1)$ & 232 & $0.2(0.06)$ & 117 & $0.14(0.03)$ & 0.103 \\
\hline Epinephrine (mg/kg) & $135(38.6)$ & $215(61.4)$ & 135 & $0.01(0.01)$ & 132 & $0.01(0.01)$ & 3 & $0.00(0.02)$ & 0.791 \\
\hline Vasopresin (mcg/kg) & $6(1.7)$ & $344(98.3)$ & 6 & $0.0(0.1)$ & 6 & $0.1(0.1)$ & 0 & 0.00 & NA \\
\hline ICU EBL (cc/kg) & 331 (94.6) & $19(5.4)$ & 331 & $30.0(35.0)$ & 219 & $38.0(47.0)$ & 112 & $24.0(16.5)$ & 0.858 \\
\hline Intubation (days) ${ }^{\#}$ & $245(70.0)$ & $105(30.0)$ & 346 & $2.0(4.0)$ & 229 & $3.0(3.00)$ & 117 & $0.0(0.0)$ & $<0.0001$ \\
\hline
\end{tabular}

Note. ${ }^{a} 0 \mathrm{~s}$ in variables are excluded. \# $0 \mathrm{~s}$ were NOT Excluded (Intubation Days)

${ }^{b} P$-values are determined from median regression for Mannitol, Amount, and Epi; Poisson regression for Intubation days, and Log-Normal regression for other continuous variables. Extubation group (IE + EE vs. DE) was used as the main predictor adjusting for age, weight, and length as covariates.NAs are due to limited sample size. $P<0.05$ are in bold character 
Table 7 Case Types

\begin{tabular}{ll}
\hline Case types for IE and EE & Number \\
\hline Atrial septal defect repair & 65 \\
Ventricular septal defect repair & 58 \\
Fontan & 43 \\
Bidirectional cavopulmonary anastomosis & 41 \\
Right ventricular outflow tract reconstruction & 40 \\
(pulmonary valve replacement) & \\
Tetralogy of Fallot repair & 30 \\
Repair atrioventricular canal, partial or complete & 18 \\
Pulmonary artery reconstruction & 12 \\
Sub-aortic membrane resection & 11 \\
Mitral valvuloplasty or replacement & 10 \\
Repair anomalous coronary artery & 9 \\
Repair Anomalous pulmonary venous return, total or partial & 4 \\
Central shunt or Blalock-Taussig shunt & 4 \\
Aortic Valve Replacement & 3 \\
Tricuspid valvuloplasty & 3 \\
Double chambered right ventricle repair & 3 \\
Aortic arch reconstruction & 3 \\
Pulmonary artery banding & 2 \\
Rastelli procedure & 2 \\
Septal myomectomy & 1 \\
Repair Cor triatriatum & 1 \\
Ross-Konno & 1 \\
DORV repair & 1 \\
& 2 \\
\hline
\end{tabular}

lower LOS in the ICU and the hospital. There was also a strong inverse relationship between younger age, smaller size, longer $\mathrm{CPB}$ time, longer aortic cross clamp time, use of deep hypothermia, longer RLP time, DHCA, need for epinephrine or vasopressin, ICU blood loss and the ability to achieve IE or EE. These variables are also markers for more complex repairs, which is more likely to lead to inadequate hemostasis or hemodynamic instability. Higher doses of fentanyl and midazolam were also inversely correlated with IE/EE.

The two main criteria our institution utilizes for IE is hemodynamic stability, without the need for escalating doses of inotropic support and minimal blood loss (less than $10 \mathrm{cc} / \mathrm{kg} / \mathrm{hr}$ ). Many pediatric patients undergoing cardiac surgery for congenital heart disease fit this criterion, especially patients over age one. Our standard protocol is to wean from CPB with milrinone; epinephrine is used if this is inadequate. Our data indicates that the need and dose of epinephrine is greater in the DE group. With respect to blood loss, there is a significantly greater amount in the DE group. With respect to transfusion requirements, the only difference between the groups was the amount of PRBC. The differences in the amount of
Table 8 Case Types

\begin{tabular}{ll}
\hline Case Types for DE & Number \\
\hline Tetralogy of Fallot repair & 31 \\
Ventricular septal defect repair & 25 \\
Aortic arch reconstruction & 24 \\
Arterial switch operation & 23 \\
Stage 1 Norwood & 21 \\
Central shunt or Blalock-Taussig shunt & 21 \\
Repair anomalous pulmonary venous return, total or partial & 14 \\
Repair atrioventricular canal & 13 \\
Damus-Kaye-Stansel procedure & 12 \\
Right ventricular outflow tract reconstruction & 11 \\
(pulmonary valve replacement) & \\
Mitral valvuloplasty or replacement & 10 \\
Bidirectional cavopulmonary anastamosis & 10 \\
Truncus arteriosus repair & 8 \\
Unifocalization & 8 \\
Double outlet right veneticle repair & 5 \\
Pulmonary artery reconstruction & 5 \\
Aortic valve replacement & 4 \\
Repair anomalous coronary artery & 2 \\
Ross-Konno & 4 \\
Tricuspid valvuloplasty & 2 \\
Fontan & 4 \\
Subrial septectomy & 1 \\
& 2 \\
\hline
\end{tabular}

other component blood products was not significant, which was surprising to the authors. It is noteworthy, that inadequate hemostasis is one of the main reasons patients at our institution do not get extubated in the OR.

Over half the patients in our analysis were infants and they represented the majority of the patients in the $\mathrm{DE}$ group. However, even one third of this cohort were in the IE/EE groups, including patients undergoing complex repairs. If hemodynamic stability and adequate hemostasis can be achieved in this cohort, they can be candidates for IE. The median age of the patients in this infant DE group was 20 days, significantly different than the roughly six months ages of the IE/EE cohort. However, even neonates experienced IE or EE in our data set, but we did not do a sub-analysis of neonates alone since this cohort was too small. Other investigators have also demonstrated this association between age and the ability to IE or EE. In a retrospective review of over 900 pediatric patients undergoing surgery for congenital heart disease, there was an inverse relationship between patient age and the ability to achieve EE, i.e., the younger the patient the less likely they were to experience EE [15]. Other investigators have reported similar results [16]. 
One noteworthy meta-analysis demonstrated that various investigators define early extubation differently [17]. Early extubation ranged from in the operating room to as much as $24 \mathrm{~h}$ after surgery. The anesthetic protocols also varied greatly between the studies, with varying inhalational agents, drugs and doses. Modern, short-acting anesthetics, like remifentanil, dexmedetomidine and propofol and various regional techniques, like intrathecal or caudal morphine have made early and immediate extubation possible, even for longer surgeries involving complex repairs [18]. Sugammadex has been another noteworthy addition to the anesthetic armamentarium, but more studies need to be conducted in this patient population.

Our institutional anesthetic protocol is as follows: Induction is by mask with sevoflurane if no indwelling IV is present and usually with Propofol $(1-2 \mathrm{mg} / \mathrm{kg})$ if an indwelling IV catheter is present. Etomidate or fentanyl is substituted for propofol if the physiology dictates that myocardial depression or vasodilatation should be avoided. Maintenance is with dexmedetomidine $(0.5-1 \mathrm{mcg} / \mathrm{kg} / \mathrm{hr})$, propofol (50-100 $\mathrm{mcg} / \mathrm{kg} / \mathrm{min})$, rocuronium and sevoflurane or some combination, coupled with the ONQ Pain Buster and more recently liposomal bupivacaine for postoperative analgesia $(4 \mathrm{mg} / \mathrm{kg}$ liposomal bupivacaine admixed with $3 \mathrm{~mL} 0.25 \%$ bupivacaine for every $\mathrm{mL}$ of liposomal bupivacaine, plus $0.9 \%$ NS to produce a volume of $10 \mathrm{~mL}$ per linear inch of incision plus $10 \mathrm{~mL}$ for every chest tube site). Sugammadex is now routinely used for reversal of the neuromuscular block from rocuronium. Fentanyl doses are routinely kept below $5 \mathrm{mcg} / \mathrm{kg}$ in those patients where IE/EE is to be attempted; higher doses of fentanyl greatly reduce the possibility of IE/EE. Adequacy of reversal of neuromuscular blockage is assessed with a peripheral nerve stimulator at the conclusion of the case. Patients are allowed to breath spontaneously and extubation occurs when the breathing pattern is regular and tidal volumes exceed $5 \mathrm{~mL} / \mathrm{kg}$. The airway is assessed for obstruction before leaving the OR. If airway obstruction exists it is treated with airway positioning or a nasal airway. A ROTEM based transfusion protocol is utilized for hemostasis; the largest reason for delayed extubation is inadequate hemostasis.

\section{Conclusions}

Older and larger patients were more likely to experience IE. Length of cardiopulmonary bypass, aortic cross-clamp time, use of deep hypothermia, and length of regional low flow perfusion were inversely correlated with the incidence of IE. The need for epinephrine or inotropes other than milrinone were inversely correlated with IE. Greater blood loss and need for transfusion with packed red blood cells was inversely associated with IE. IE can be successfully accomplished in a majority of pediatric patients undergoing surgery for congenital heart disease, including in a minority of infants.

\section{Abbreviations}

CHD: Congenital heart disease; CPB: Cardiopulmonary bypass;

CPS: Cardiopulmonary support; Cryo: Cryoprecipitate; CS: Cell saver; DE: Delayed extubation; DHCA: Deep hypothermic circulatory arrest; EBL: Estimated blood loss; EE: Early extubation; ES-RO: End of surgery to room out; FFP: Fresh frozen plasma; HFC: Human fibrinogen concentrate; ICU: Intensive care unit; IE: Immediate extubation; IQR: Interquartile range; IRB: Institutional review board; LOS: length of stay; OR: Operating room; Plat: Platelets; PRBC: Packed red blood cells; RLF: Regional low flow

\section{Acknowledgments}

None.

\section{Authors' contributions}

CFT: Made primary contribution to study conception and design, data acquisition and analysis and drafting and revising the manuscript for intellectual content. SO: Made substantial contribution to study conception and design, data acquisition and drafting and revising the manuscript for intellectual content. RGL: Made substantial contribution to data acquisition and drafting and revising the manuscript for intellectual content. DS: Made substantial contributions to data analysis and drafting and revising the manuscript for intellectual content. WW: Made substantial contributions to data analysis and drafting and revising the manuscript for intellectual content. JH: Made substantial contribution to data acquisition and drafting and revising the manuscript for intellectual content. MI: Made substantial contribution to data acquisition and drafting and revising the manuscript for intellectual content. RB: Made substantial contribution to drafting and revising the manuscript for intellectual content. All authors read and approved the final manuscript.

\section{Funding}

None.

Availability of data and materials

The datasets used and/or analyzed during the current study are available from the corresponding author on reasonable request.

Ethics approval and consent to participate

This study received Institutional Review Board (IRB) exempt status from the Research Institute of Nicklaus Children's Hospital.

Consent for publication

Not applicable.

\section{Competing interests}

The authors declare that they have no competing interests.

\section{Author details}

${ }^{1}$ Cardiac Anesthesia, Department of Anesthesiology, Nicklaus Children's Hospital, Miami, USA. ${ }^{2}$ Research Institute, Nicklaus Children's Hospital, Miami, USA. 'Division of Cardiovascular Surgery, Nicklaus Children's Hospital, Miami, USA.

Received: 8 July 2019 Accepted: 2 January 2020

Published online: 06 January 2020

\section{References}

1. Hoffman JL, Kaplan S. The incidence of congenital heart disease. J Am Coll Cardiol. 2002;39(12):1890-900.

2. Robinson A. Early extubation after pediatric heart surgery: the future? Crit Care Med. 2002;30:940-1

3. Prakanrattana U, Valairucha S, Sriyoschati S, Pornvilawan S, Phanchaipetch T. Early extubation following open heart surgery in pediatric patients with congenital heart diseases. J Med Assoc Thail. 1997;80:87-95.

4. Fischer JE, Allen P, Fanconi S. Delay of extubation in neonates and children after cardiac surgery: impact of ventilator-associated pneumonia. Intensive Care Med. 2000;26:942-9.

5. Schuller JL, Bovill JG, Nijveld A, Patrick R, Marcelletti C. Early Extubation of the trachea after open heart surgery for congenital heart disease. $\mathrm{Br} J$ Anaesth. 1984;56:1101-8. 
6. Barash PG, Lescovich F, Katz JD, Talner NS, Stansel HC. Early extubation following pediatric cardiothoracic operation: a viable alternative. Ann Thorac Surg. 1980;29(3):228-33.

7. Heard GG, Lamberti JJ, Park SM, Waldman JD, Waldman J. Early Extubation after surgical repair of congenital heart disease. Crit Care Med. 1985;13(10): 830-2.

8. Heinle JS, Fox LS. Early extubation of neonates and young infants after cardiac surgery. Semin Thorac Cardiovasc Surg Pediatr Card Annu. 1998;1: 103-8.

9. Bennyworth BD, Mastropietro CW, Graham EM, Klugman D, Costello JM, Zhang W, Gaies M. Variation in extubation failure rates after neonatal congenital heart surgery across Pediatric Cardiac Critical Care Consortium hospital. J Thorac Cardiovasc Surg. 2017;153(6):1519-26.

10. Harris KC, Holowachuk S, Pitfield S, Sanatani S, Froese N, Potts JE, Gandhi SK. Should early extubation be the goal for children after congenital cardiac surgery? J Thorac Cardiovasc Surg. 2014;148:2642-8.

11. Varghese J, Kutty S, Abdullah I, Hall S, Shostrom V, Hammel J. Preoperative and intraoperative predictive factors of immediate extubation after neonatal cardiac surgery. Ann Thorac Surg. 2016;102:1588-9.

12. Mahle WT, Jacobs JP, Jacobs ML, Kim S, Kirshbom PM, Pasquali SK, Austin EH, Kanter KR, Nicolson SC, Hill KD. Early extubation after repair of tetralogy of fallot and the fontan procedure: an analysis of the society of thoracic surgeons congenital heart surgery database. Ann Thorac Surg. 2016;102: 850-8.

13. Varghese J, Kutty S, Moukagna KSB, Craft M, Abdullah I, Hammel JM. Fiveyear experience with immediate extubation after arterial switch operations for transposition of great arteries. Euro J Cardio Thorac Surg. 2017;51:72834

14. Halimic M, Dinarevic SM, Begic Z, Kadic A, Pandur S, Omerbasic E. Early extubation after congenital heart surgery. J Health Sci. 2014:4(3):156-61.

15. Neirotti RA, Jones D, Hackbarth R, Paxson-Fosse G. Early extubation in congenital heart surgery. Heart Lung Circ. 2002;11(3):157-61.

16. Davis $S$, Worley $S$, Mee RBB, Harrison AM. Factors associated with early extubation after cardiac surgery in young children. Pediatr Crit Care Med. 2004:5(1):63-8.

17. Alghamdi AA, Singh SK, Hamilton BCS, Mus M, Yadava M, Holtby H, Van Arsdell GS, Al-Radi OO. Early extubation after pediatric cardiac surgery: systematic review, meta-analysis, and evidence-based recommendations. J Card Surg. 2010;25(5):586-95.

18. Mittnacht AJ, Hollinger I. Fast-tracking in pediatric cardiac surgery - the current standing. Ann Card Anaesth. 2010;13:92-10.

\section{Publisher's Note}

Springer Nature remains neutral with regard to jurisdictional claims in published maps and institutional affiliations.

Ready to submit your research? Choose BMC and benefit from:

- fast, convenient online submission

- thorough peer review by experienced researchers in your field

- rapid publication on acceptance

- support for research data, including large and complex data types

- gold Open Access which fosters wider collaboration and increased citations

- maximum visibility for your research: over $100 \mathrm{M}$ website views per year

At BMC, research is always in progress.

Learn more biomedcentral.com/submissions 